\title{
Purely endoscopic pterional extradural approach: A novel technique for repair of cerebrospinal fluid rhinorrhea
}

\author{
Ajit Kumar Sinha, Sumit Goyal \\ Department of Neurosurgery, Sir Ganga Ram Hospital, New Delhi, India
}

\begin{abstract}
Study Design: Retrospective descriptive study of an innovative surgical technique. Objective: To assess the feasibility and success of repair of transfrontal sinus cerebrospinal fluid (CSF) rhinorrhea through pterional transcranial extradural approach using endoscope. Summary of Background Data: Repair of CSF rhinorrhea has seen advancement with the evolution of endoscopic transnasal techniques. However, leaks from defect in the posterior wall of frontal sinus still remain a challenge for the skull base surgeons and requires conventional craniotomy more often. We describe a novel technique to repair these leaks by purely endoscopic pterional extradural (PEPE) approach thereby avoiding complications associated with conventional craniotomy and endoscopic transnasal approaches. Materials and Methods: Thirty-five patients with traumatic CSF rhinorrhea from the posterior wall of frontal sinus underwent repair with the present technique. They were followed up for 6-18 months and were evaluated for feasibility of procedure, recurrence of leak, and occurrence of the fresh neurological deficit. Results: Thirty-five patients underwent CSF rhinorrhea repair using the above technique. The procedure was accomplished in all patients without any intraoperative complications. There was no requirement of blood transfusion in any case. All patients had a cessation of CSF leak in the postoperative period, and there was no recurrence. There was no evidence of frontal lobe retraction injury in any of these patients, and no fresh neurological deficit was observed. Conclusion: This PEPE approach to repair CSF leak through the posterior wall of the frontal sinus is a novel technique in which we can avoid disadvantages associated with both conventional craniotomy as well as transnasal endoscopic approaches.
\end{abstract}

Key words: Cerebrospinal fluid rhinorrhea, endoscopic repair, frontal sinus leak, transcranial repair

\section{Introduction}

Cerebrospinal fluid (CSF) rhinorrhea is defined as the leakage of CSF from the nose due to communication with the subarachnoid space and may be classified as traumatic and nontraumatic. The most common anatomic sites of the defect are the areas of congenital weakness of anterior cranial fossa. ${ }^{[1,2]}$

\section{Address for correspondence:}

Dr. Ajit Kumar Sinha, Department of Neurosurgery,

Sir Ganga Ram Hospital, New Delhi - 110 060, India.

E-mail: ajitalkasinha@yahoo.co.uk

\begin{tabular}{|l|l|}
\hline \multicolumn{2}{|c|}{ Access this article online } \\
\hline Quick Response Code: & Website: \\
\hline & www.ruralneuropractice.com \\
\cline { 2 - 3 } & \\
\hline & DOI: \\
\hline
\end{tabular}

These leaks of the sinonasal cavity are repaired using accepted endoscopic techniques, with relatively high success rates. ${ }^{[3]}$ However, frontal sinus is technically the most demanding area of the sinonasal cavity, and further, the leaks from the posterior table of the frontal sinus push the limit of the endoscopic repair. In most of these techniques, the frontal sinus is obliterated leading to the possibility of mucocele formation. Further, there is difficulty in the visualization of the full bony defect and impossible to put an underlay graft (between the bone and the dura) which is most desired in these repairs.

This is an open access article distributed under the terms of the Creative Commons Attribution-NonCommercial-ShareAlike 3.0 License, which allows others to remix, tweak, and build upon the work non-commercially, as long as the author is credited and the new creations are licensed under the identical terms.

For reprints contact: reprints@medknow.com

How to cite this article: Sinha AK, Goyal S. Purely endoscopic pterional extradural approach: A novel technique for repair of cerebrospinal fluid rhinorrhea. J Neurosci Rural Pract 2016;7:310-3. 
Therefore, in these cases, we are left with traditional unifrontal or bifrontal craniotomy, which is associated with increased morbidity due to frontal lobe retraction and the scar, is not esthetically appealing.

We describe our experience of repair of CSF leak from the posterior table of a frontal sinus using our novel technique of purely endoscopic pterional extradural (PEPE) approach using temporalis fascia or fascia lata.

\section{Materials and Methods}

All patients admitted to our hospital with a diagnosis of CSF leak from the defect in the posterior table of frontal sinus from January 2012 to December 2014 underwent repair using this novel technique.

All surgeries were performed under general anesthesia, and the lumbar drain was not placed in any of these patients. The head is extended by $15^{\circ}$ and is $60^{\circ}$ rotated to the other side to allow the brain to fall and to get comfortable trajectory with the endoscope.

Ipsilateral curved temporofrontal incision of around $6 \mathrm{~cm}$ in length is given just in front of the hairline. The galea is cut along with the temporalis fascia in the same line and is retracted using fish hooks or self-retaining retractor. An approximately $2 \mathrm{~cm} \times 2 \mathrm{~cm}$ pterional craniotomy is then made using a high-speed drill with the lower border of craniotomy flush with the lesser wing of sphenoid. There is no necessity of entering the temporal side. The dura is then progressively and gradually stripped from orbital roof under constant visualization of $0^{\circ}$ endoscope (Karl Storz, Germany). The direction of dissection is from posterolateral to anteromedial guided by the left-hand finger placed at the lower end of glabella. Though neuronavigation can also be used to guide the direction of dissection, the need was not felt by the authors in any of these cases.

Initially, the endoscope advancement gives a featureless picture without much anatomical landmarks, however, toward the medial side, we start visualizing the bony defect in the sinus more clearly. On further stripping of the dura, the full extent is visualized and defined. The herniating neural tissues are then reduced. Any epidural bleed if encountered is stopped by using floseal hemostatic matrix (Baxter, Deerfield, IL, USA). The defect is then defined using $30^{\circ}$ and $45^{\circ}$ endoscope to avoid further retraction [Figure 1]. After the defect is fully defined the temporalis fascia is harvested (fascia lata may also be used) and is secured over the defect using fibrin sealant [Figure 2]. After achieving hemostasis, the brain

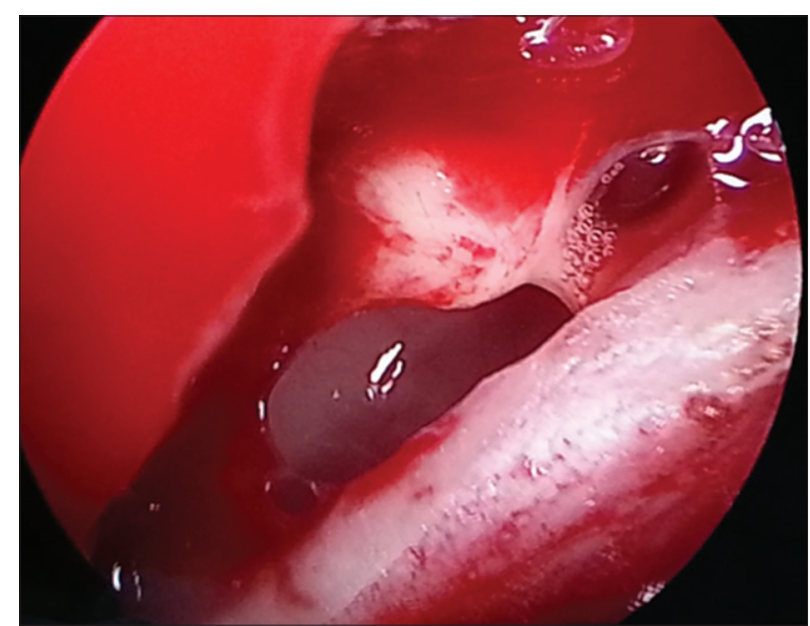

Figure 1: Intraoperative photograph showing the completely delineated defect in posterior table of frontal sinus after extradural dissection

is allowed to fall back by elevating the head by $30^{\circ}$. The bone flap is then secured back with titanium miniplates, and the wound is closed in layers.

\section{Results}

Thirty-five patients with posttraumatic CSF rhinorrhea were repaired with the present technique. There were 23 males and 12 females with age ranging from 8 years to 53 years (mean age was 25.4 years). The bilateral defect was present in 5 patients while 30 patients had unilateral defect [Figure 3] in the posterior wall of the frontal sinus. These patients had history of clear watery nasal discharge with the presence of glucose in the discharge fluid and were radiologically confirmed by computed tomography (CT) cisternography or brow down magnetic resonance imaging showing herniation of brain tissue. All these patients had a failure of earlier conservative management. The duration of persistent CSF leak varied for a period of 4 weeks to 25 weeks with mean of 14.5 weeks and median of 18 weeks. Clinical follow-up was for a period of 6-18 months with a mean follow-up of 12.3 months.

There was no intraoperative complication in any of these procedures. The mean surgical time after anesthesia was $95 \mathrm{~min}$. Blood transfusion was not required in any of the procedures. Lumbar drain was not placed in any of the patients during the surgery or during the postoperative period. The size of bony defect varied from $2 \mathrm{~mm}$ to $12 \mathrm{~mm}$ with a mean defect of $5 \mathrm{~mm}$. Average craniotomy site was $2 \mathrm{~cm}$ in diameter.

All patients had a cessation of CSF leak, and there was no recurrence during the follow-up period. There was no fresh neurological deficit. Seven patients had 
preoperative anosmia. However, olfaction was preserved in the remaining 28 patients in the postsurgical period.

Postprocedure CT scan was routinely done after $48 \mathrm{~h}$ which did not reveal any insult to the frontal lobe due to retraction [Figure 4].

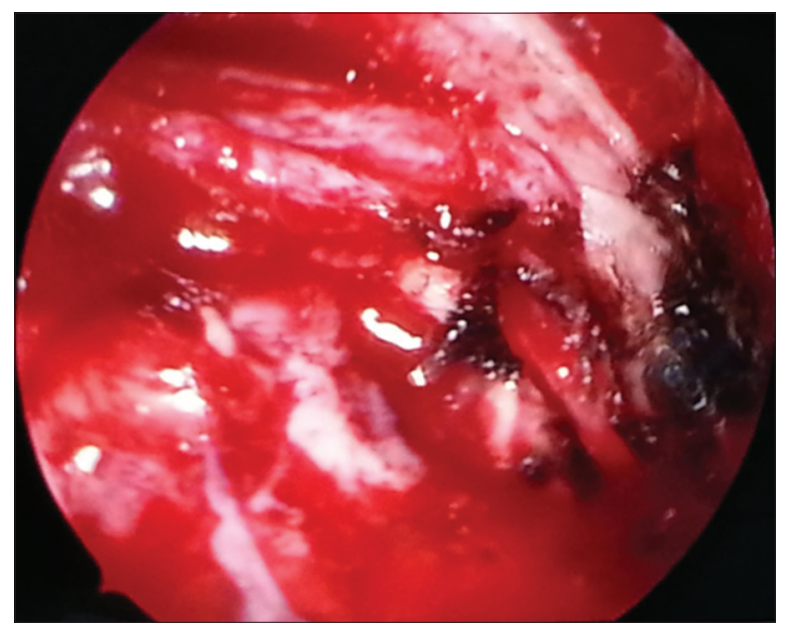

Figure 2: Intraoperative photograph after covering the defect with temporalis fascia graft

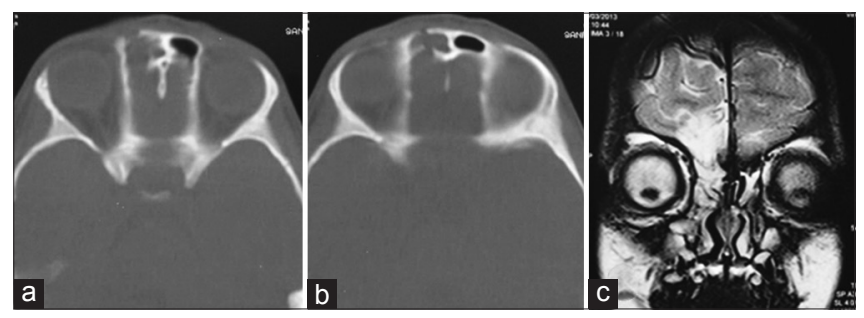

Figure 3: ( $\mathrm{a}$ and $\mathrm{b})$ Computed tomography scan bone window of a patient with posttraumatic cerebrospinal fluid rhinorrhea showing fracture of the frontal sinus, (c) magnetic resonance imaging of the same patient showing herniation of brain tissue through the defect in posterior wall of frontal sinus with gliosis in the adjacent frontal lobe

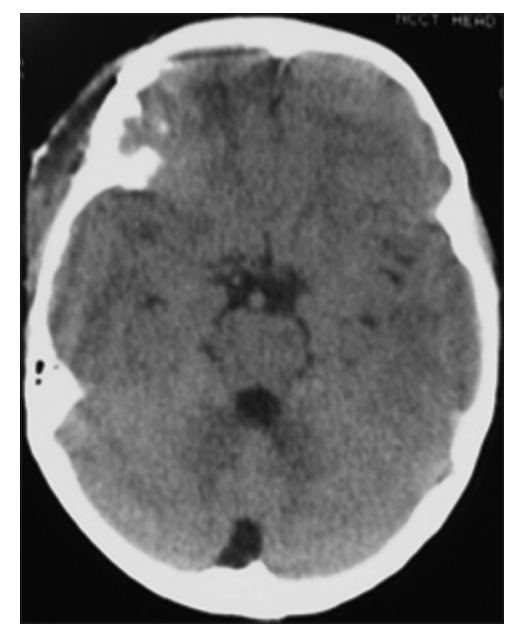

Figure 4: Immediate postoperative computed tomography scan of the patient who underwent repair of cerebrospinal fluid leak through purely endoscopic pterional extradural approach did not show any retractional injury to frontal lobe

\section{Discussion}

CSF leak repair remains one of the challenging surgeries for the skull base surgeons. The route of CSF leak is decided by the location of injury in relation to bone and underlying meninges. The management of CSF leak has improved since the development of nasal endoscopy technique, availability of fibrin glue, better fistula localization, and availability of three-dimensional imaging techniques. ${ }^{[4]}$

Despite the advancement in the technique of repair, a leak from the posterior wall of frontal sinus especially extending laterally still remains a daunting task via the transnasal endoscopic approach and usually requires an open approach. ${ }^{[5,6]}$

The extracranial techniques used to repair these defects require obliteration of frontal sinus leading to mucocele formation, besides the difficulty in defining the full extent of the defect especially lateral and posterior, which in turn requires a lot of mutilating bone work. ${ }^{[5]}$ Such mutilating bone work is not acceptable in children.

Traditionally, a coronal incision with obliteration of the sinus is used which is associated with morbidity due to frontal retraction, anosmia, and alteration of frontal contour leading to cosmetic deformity. ${ }^{[7,8]}$

The present technique which the authors describe is a novel technique in which the optical advantages of the endoscope are utilized to access the inaccessible areas through the pterional mini-craniotomy and offers many advantages when compared to traditional unifrontal or bifrontal craniotomy. The above procedure can also be accomplished through a burr hole, but it would be cosmetically inferior when compared to a $2 \mathrm{~cm}$ minicraniotomy which leaves no bone defect.

With the present technique, all the above-mentioned complications can be avoided as the surgical corridor is made through a small incision, without disturbing the frontal contour, working extradurally with minimal retraction of the ipsilateral frontal lobe. The chosen trajectory though longer, avoids the problem in visualization due to the orbital roof convexity and the complete defect can be visualized and defined. Moreover, as the frontal sinus is not opened in this approach, there is no need to obliterate the sinus thereby maintaining the sinus patency. This procedure can be technically also called as transcranial extradural frontal sinoplasty.

However, the need of less invasive procedure is well felt, and other techniques are reported to overcome 


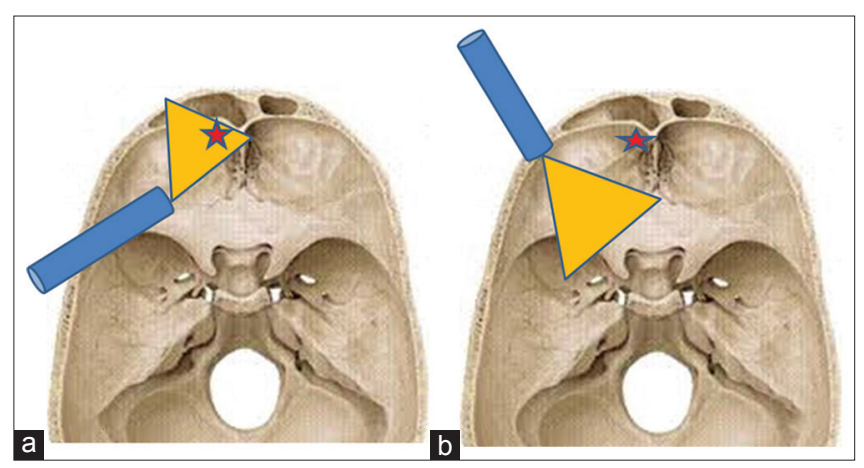

Figure 5: Diagram showing the advantage of using a pterional approach: (a) With $30^{\circ}$ endoscope which allows a more direct and complete visualization of the posterior wall of frontal sinus when compared to the (b) supraorbital approach with $0^{\circ}$ endoscope. Asterix indicates the site of leak

large craniotomies. ${ }^{[9]}$ Das and Balasubramanian, ${ }^{[10]}$ also described a novel technique of repair of such defects using external frontal sinusotomy, in which the authors drill two holes in the anterior wall of the frontal sinus. The disadvantage of which is a facial scar and the opening of frontal sinus which is avoided in our technique.

The limitation of the present technique is bilateral repair from one side. Although bilateral repair is performed in five of our patient, it was done as a separate procedure on other side after 2 weeks. We feel that approaching the bilateral leak from one side may lead to opposite side dural tear and injury to frontal lobe or olfactory nerve in the process. Furthermore, as compared to lateral supraorbital keyhole approach, our approach has a longer trajectory, but the complete visualization of the defect in sinus wall and its repair is much easier due to the peculiar anatomy of the anterior cranial fossa [Figure 5].

Thus by using PEPE approach successful repair of CSF leak from the posterior table of the frontal sinus is achieved and to the best of our knowledge, this novel technique has not been reported in the literature before.

\section{Conclusion}

Surgical treatment of CSF rhinorrhea due to leak from the posterior table of the frontal sinus is a difficult task to be performed and still requires a conventional craniotomy and obliteration of the sinus for repair.

We describe a new technique of successful repair of such leaks by a PEPE approach in which we can avoid disadvantages associated with conventional craniotomy and transnasal endoscopic approaches. The initial results of this technique are encouraging and can potentially be extended to repair other skull base defects also.

\section{Financial support and sponsorship \\ Nil.}

\section{Conflicts of interest}

There are no conflicts of interest.

\section{References}

1. Tolley NS, Lloyd GA, Williams HO. Radiological study of primary spontaneous CSF rhinorrhoea. J Laryngol Otol 1991;105:274-7.

2. Stankiewicz JA. Cerebrospinal fluid fistula and endoscopic sinus surgery. Laryngoscope 1991;101:250-6.

3. Mattox DE, Kennedy DW. Endoscopic management of cerebrospinal fluid leaks and cephaloceles. Laryngoscope 1990;100:857-62.

4. Hegazy HM, Carrau RL, Snyderman CH, Kassam A, Zweig J. Transnasal endoscopic repair of cerebrospinal fluid rhinorrhea: A meta-analysis. Laryngoscope 2000;110:1166-72.

5. Woodworth BA, Schlosser RJ, Palmer JN. Endoscopic repair of frontal sinus cerebrospinal fluid leaks. J Laryngol Otol 2005;119:709-13.

6. Roehm CE, Brown SM. Unilateral endoscopic approach for repair of frontal sinus cerebrospinal fluid leak. Skull Base 2011;21:139-46.

7. Marshall AH, Jones NS. Cerebrospinal fluid rhinorrhoea. In: Gleeson M, Jones NS, Burton MJ, Clarke R, Browning G, Luxon L, editors. Scott-Browns's Otorhinolaryngology, Head and Neck Surgery. London: Edward Arnold; 2008. p. 1636-44.

8. Aarabi B, Leibrock LG. Neurosurgical approaches to cerebrospinal fluid rhinorrhea. Ear Nose Throat J 1992;71:300-5.

9. Lasisi OA, Ahmad BM, Ogunbiyi OA. Trans-frontal extracranial approach in repair of cerebrospinal fluid fistula. Afr J Med Med Sci 2006;35:169-71.

10. Das PT, Balasubramanian D. External frontal sinusotomy and endoscopic repair of cerebrospinal fluid fistula in the posterior wall: Preliminary report of a new technique. J Laryngol Otol 2011;125:802-6. 